\title{
RETRACTED ARTICLE: From the human visual system to the computational models of visual attention: a survey
}

\author{
Sílvio Filipe • Luís A. Alexandre
}

Published online: 9 January 2013

(C) Springer Science+Business Media Dordrecht 2013

This article has been retracted by the authors. The article included text and ideas taken by the first author, without acknowledgement, from the following published article: "State-ofthe-art in visual attention modeling", Ali Borji, Laurent Itti, IEEE Transactions on Pattern Analysis and Machine Intelligence 35(1) (2013) 185-207, published online 05/04/12. Most notably:

- In Sect. 3.1 (Biological plausible methods) the following paragraphs or sentences largely derive from the Borji and Itti article: "Rosenholtz (1999), Rosenholtz et al. (2004) designed a model ..."; "In Gu et al. (2005), a saliency map ... "; "Le Meur et al. (2006) proposed ..."; "Kootstra et al. (2008) developed ..."; "Marat et al. (2009) proposed ..."; "Chikkerur et al. (2010) proposed ..."; and "Murray et al. (2011) introduced ...".

- In Sect. 3.2 (Computational methods) the following paragraphs or sentences largely derive from the Borji and Itti article: "Salah et al. (2002) proposed ..."; "Ramström and Christensen (2002) introduced ..."; "In Rao et al. (2002) and Rao (2005), they proposed ..."; "Jodogne and Piater (2007) introduced ..."; "Boccignone (2008) presented ..."; "Rosin (2009) proposed ..."; "Mahadevan and Vasconcelos (2010) presented ..."; and "Wang et al. (2011) introduced ...".

- In Sect. 3.3 (Hybrid methods) the following paragraphs or sentences largely derive from the Borji and Itti article: "Lee and Yu (1999) proposed ..."; "Peters et al. (2005), Peters and Itti (2007a,b, 2008) trained ..."; "Weights between two nodes ..."; "The model consists of a nonlinear ..."; "Zhang et al. (2007, 2008) proposed ..."; "Pang et al. (2008) presented ..."; "Zhang et al. (2009) extended ..."; and "Li et al. (2010a) presented ...".

- Section 6 (Discussion) largely derives from, or summarizes ideas presented in, Sects. 2.1, 2.2, 2.4, 2.6 and 3.1-3.8 of the Borji and Itti article.

The first author apologizes for his action.

\footnotetext{
S. Filipe $(\varangle) \cdot$ L. A. Alexandre

IT, Instituto de Telecomunicações, SOCIA, Soft Computing and Image Analysis Group, Department of Computer Science, University of Beira Interior, 6200-001 Covilhã, Portugal e-mail: sfilipe@ubi.pt

L. A. Alexandre

e-mail: 1fbaa@di.ubi.pt
} 